\title{
5. Mass Influx Obtained From Low-Light-Level Television Observations of Faint Meteors
}

\author{
Robert J. Naumann \\ K. Stuart Chifton \\ George C. Marshall Space Flight Center \\ Huntsville, Alabama
}

\begin{abstract}
Since the advent of low-light-level television (LLLTV) systems, it has been recognized that such devices offer the ability to observe meteors as faint as 10th magnitude which allows the extension of optical meteor data to masses as small as $10^{-4}$ gram. The Space Sciences Laboratory at Marshall Space Flight Center has been actively engaged in such observations using image orthicons and intensified SEC vidicons.

The results of these observations are presented along with an interpretation in terms of mass-flux. This interpretation includes the development of a relationship between peak luminosity of a meteor and mass, velocity, and zenith angle that was derived from single body meteor theory and compares favorably with results obtained from the Artificial Meteor Program. Also included in the mass-flux interpretation is an analysis of the observation response of a LLLTV system to fixed and moving point sources.
\end{abstract}

$\mathrm{T}$ HE PRESENT MODEL of the meteoroid mass distribution is based on an extrapolation from ground-based photographic observations of the larger meteoroids with masses of the order of grams to satellite-borne penetration measurements of meteoroids with masses in the microgram range. The mass range representing the greatest damage potential to manned space vehicles is from 1 to $100 \mathrm{mg}$. The fact that the meteoroid population in this region must be inferred from an extrapolation over 6 orders of magnitude between two points that are determined by completely different properties of meteoroids through interactions that are poorly understood physically and cannot be adequately tested experimentally has caused some concern among those responsible for establishing the meteoroid environment. Also, there are legitimate scientific reasons for extending the ground-based optical measurements to fainter meteors. Of primary interest is the determination of the slope of the mass distribution curve or the population index parameter. This will greatly improve the confidence in the extrapolation as well as reduce the range over which the extrapolation must be carried out, and will provide a badly needed consistency check between the ground-based and satellite measurements.

This paper discusses the techniques and results of using LLLTV observations to determine the meteoroid mass distribution in the region from grams to milligrams.

\section{DESCRIPTION OF THE SYSTEM}

Recent developments in low-light-level television systems have allowed the observation of much fainter meteors than could be photographed. This improvement results primarily from the much higher quantum efficiency of the photodetector which results in much smaller integration 
times. This is particularly important in meteor work since it is advantageous to have the integration time shorter than the event duration. Our system consists of a Commercial Electronics camera chain using a Westinghouse WL-32000 Intensifier-SEC vidicon tube with a $105-\mathrm{mm}$ f/.75 Rayxar lens. This affords a 13 by $16^{\circ}$ field of view. The effective integration time of the system is very close to the standard frame time of $1 / 30 \mathrm{~s}$, which is ideal for meteor work.

The ultimate theoretical sensitivity for the system is $m_{v}=14.26$. This was estimated by requiring a star to produce 1 photoelectron at the photocathode per integration time. The system is invariably limited by sky background which, even under ideal conditions, is 2 orders of magnitude above dark current. Stars as faint as $m_{v}=11$ have been observed. This is close to the theoretical limit of 11.6 for a sky background of $300 m_{v}=10$ stars $/ \operatorname{deg}^{2}$ and a $S / N=5$.

For moving objects, such as meteors, this limiting magnitude would apply to meteors moving nearly parallel to the optical axis so that they remain within a resolving element for one integration time. For most meteors, the $S / N$ will be decreased because the time they contribute to a resolving element is limited by the writing speed of their image. It is estimated that if the system has an observing limit of 11 magnitude for stars, it will see all meteors brighter than $m_{v}=6.4$ and 50 percent of the meteors brighter than $m_{v}=8.15$.

\section{ANALYSIS PROCEDURES}

Even though the dynamic range of a TV image is limited, photometry of point images can still be performed over as much as 6 orders of magnitude by making use of the fact that the image spreads after it reaches saturation (Beyer et al., 1966). Thus, the amount of light associated with the image is a monotonic, if not linear, function of input. The difficulty lies in the fact that obtaining light curves from the TV monitor is a time consuming and laborious task, especially for the faint meteors. Until the special video processing systems presently being developed for this purpose are available, it will not be possible to obtain light curves on a sufficient sample of meteors to establish a good distribution.

An alternative procedure, which is less time consuming, was adopted for interim use. This consists of treating the video system as a threshold detector and simply counting those meteors that are above the detection threshold. By varying the threshold through reduction of the lens aperture setting, a cumulative distribution in peak meteor magnitude is obtained.

Figure 1 shows the results of this mode of operation during two observing periods at Climax, Colorado. The camera was oriented toward the zenith. Care was taken to program the aperture settings to assure a uniform distribution of observing time at each aperture setting throughout the night. The higher rates in the period from July 31 through August 9 may be attributed to the presence of the Perseids and the $\delta$-Aquarids during this period. The details of these data will be published separately.

Taking the area of the sky within the field of view to be $445 \mathrm{~km}^{2}$, the observed rates for the non-shower period can be expressed

$$
\log \phi=-15.352+.5053 m_{v}
$$

where $\phi$ is the observed rate of meteors (number/

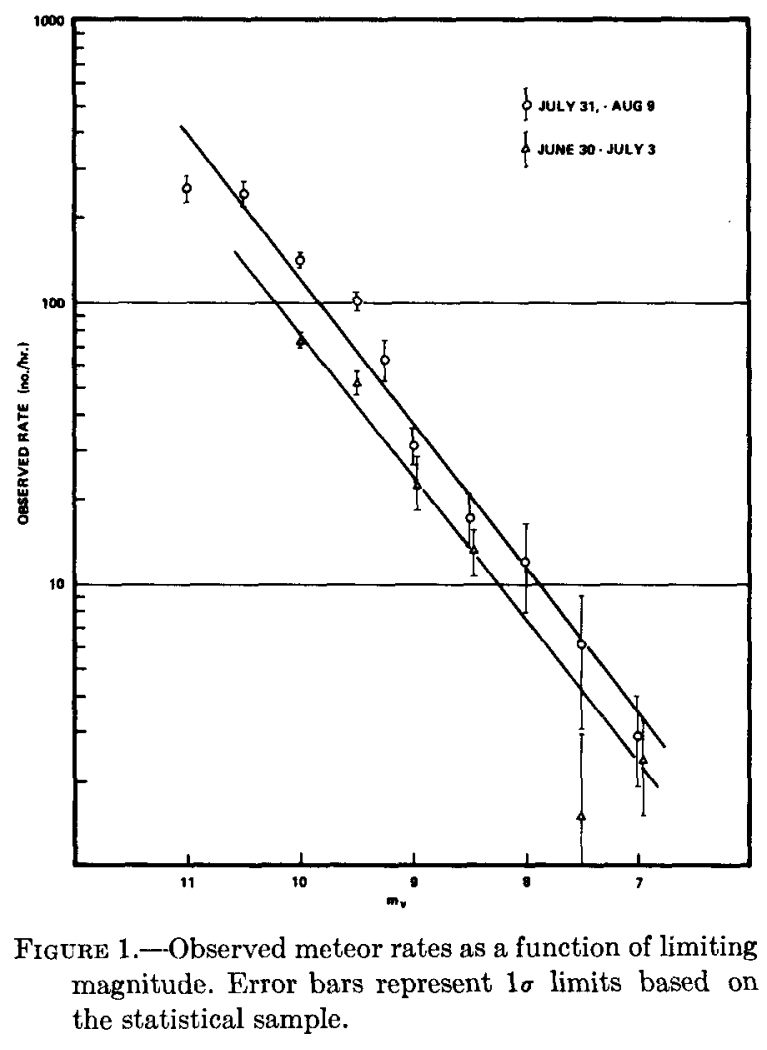


$\mathrm{m}^{2} / \mathrm{s}$ ) when $m_{v}$ is the limiting magnitude. This limiting magnitude is taken to be the magnitude of the faintest $\mathrm{AO}$ star detectable on the video screen. In a sense, this limiting magnitude represents the faintest meteor that could be detected, although practically all detectable meteors will have to be brighter than this because of their writing speed.

\section{RELATIONSHIP BETWEEN PEAK LUMINOSITY AND METEOR MASS}

A statistical analysis by Jacchia, Verniani, and Briggs (1965) of the peak brightness of meteors in terms of their mass, velocity, and entry angle yielded the result

$$
I_{P}=10^{-4.636} m_{0}^{0.9} v^{3.5}(\cos \theta)^{0.6}
$$

where $I_{P}$ is the peak intensity in units of zero magnitude stars, $m_{0}$ is the initial meteor mass in grams, $v$ is the entry velocity in $\mathrm{km} / \mathrm{s}$, and $\theta$ is the entry angle or the angle between the velocity vector and zenith.

A similar result can be derived from classical single body meteor theory by assuming that the rate of mass loss is equal to the energy input divided by the heat of vaporization $L$. In the free molecular regime,

$$
\dot{m}=-\frac{A \rho v^{3}}{2 L}=-\frac{S m^{2 / 3} \rho v^{3}}{2 L \rho_{m}{ }^{2 / 3}}
$$

where $S$ is the shape factor $(S=1.208$ for a sphere), $\rho_{m}$ is the density of the meteoroid, and $\rho$ is the atmospheric density. This neglects radiation losses, which are small compared to the heat input even at the boiling point of $\mathrm{Fe}\left(3160^{\circ} \mathrm{K}\right)$; and neglects any change in shape factor with time. Neglecting deceleration, which amounts to only a few percent velocity change in the time it takes a small meteoroid to completely burn up, and assuming an exponential atmosphere, equation (3) becomes

$$
\dot{m}=-\frac{S m^{2 / 3} v^{3}}{2 L \rho_{m}^{2 / 3}} \rho_{H} e^{-x / h}
$$

where $x=-v t \cos \theta, h$ is the scale height, and $\rho_{H}$ is the atmospheric density at $x=0$. This differential equation may be solved by separation of variables. It is convenient to define $t=0$ at meteor burnout, or $m=0$. The solution is then

where

$$
m=m_{0}\left(1-e^{\beta t}\right)^{3}
$$

$$
\beta=\frac{v \cos \theta}{h}
$$

and $m_{0}$, the initial mass, is

$$
m_{0}=\left(\frac{S v^{3} \rho_{H}}{6 L \rho_{m}^{2 / 3} \beta}\right)^{3}
$$

Differentiating the solution,

$$
\dot{m}=-3 \beta m_{0}\left(1-e^{\beta t}\right)^{2} e^{\beta t}
$$

The peak $\dot{m}$ is obtained by equating $\ddot{m}$ from equation (6) to 0 to find the time $t_{p}$ when $\dot{m}$ is maximized. This yields

$$
e^{\beta t} p=1 / 3
$$

Putting this in equation (5),

$$
\dot{m}_{p}=-4 / 9 \beta m_{0}
$$

The radiant intensity from a meteor is given by

$$
I=-\frac{\tau}{2} \dot{m} v^{2}
$$

where $\tau$ is the luminous efficiency. Using equation (7),

$$
I_{P}=\frac{\tau}{2} \frac{4}{9} \frac{m_{0} v^{3}}{h} \cos \theta
$$

The luminous efficiency for $\mathrm{Fe}$ has been determined experimentally from Trailblazer (Ayers et al., 1970) and is expressed by

$$
\tau=10^{-17.95} v(\mathrm{~cm} / \mathrm{s})
$$

Figure 2 compares the light curve obtained with this model with an observed light curve from one of the Fe Trailblazer meteors.

For stony meteors, Cook, Jacchia, and McCrosky (1963) recommend

$$
\tau=10^{-18.91} v(\mathrm{~cm} / \mathrm{s})
$$

which is consistent with estimates of Ayers, McCrosky, and Shao based on Trailblazer measurements. Putting this value in equation (9), and choosing $h=5.4 \mathrm{~km}$ which corresponds to $80 \mathrm{~km}$, results in

$$
I_{P}=10^{-5.297} m_{0} v^{4} \cos \theta
$$




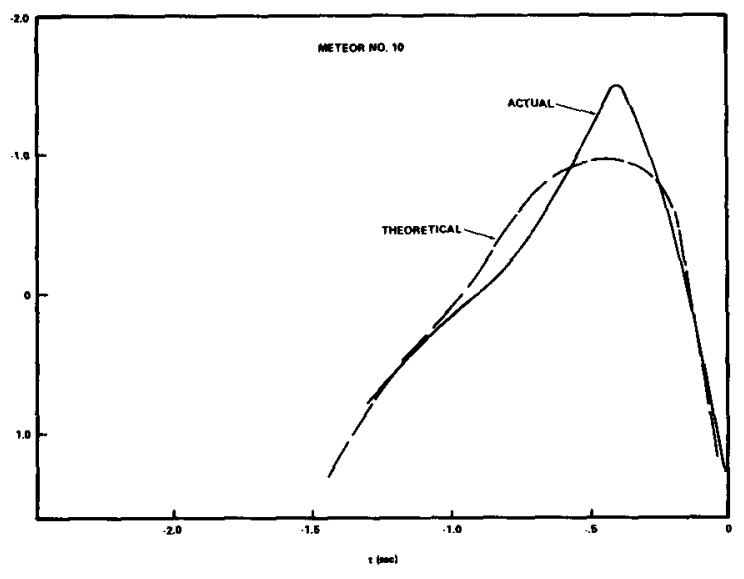

Figure 2,-Comparison of theoretical light curve compared with the measured light curve of an artificial meteor.

with the units the same as in equation (2). For the case of a $1 \mathrm{~g}$ meteor at $22 \mathrm{~km} / \mathrm{s}$, typical of cases from which equation (2) was obtained,

$$
I_{P}= \begin{cases}1.154 & \text { (eq. 2) } \\ 1.182 & \text { (eq. 12) }\end{cases}
$$

The fact that the simple theory yields almost identical results as the empirical approach, together with the desirability of having some theoretical basis for determining the functional relationship of luminous intensity with mass, velocity, and entry angle, are the bases for choosing equation (12) as the functional observing relationship for the analysis.

\section{SYSTEM RESPONSE TO MOVING TARGETS}

As was stated previously, the detection threshold of a moving object is increased because the photons are spread over a number of resolving elements instead of contributing to the signal in a single element. A first order attempt to derive the system response to moving targets is to simply require that the minimum detectable moving object deliver the same number of photons during the time it resides in a resolving element as a minimum detectable stationary object in one integration time. The residence time is

$$
t=\frac{A_{r}^{1 / 2}}{F \omega}
$$

where $A_{r}$ is the area of a resolving element, $F$ is the focal length of the lens, and $\omega$ is the angular rate.

The criterion for detectability is

$$
I_{L} \frac{A_{r}^{1 / 2}}{F \omega}=I_{T} \tau
$$

where $I_{T}$ is the threshold intensity for stationary objects, $I_{L}$ is the limiting intensity for moving objects, and $\tau$ is the integration time. The photocathode is $40 \mathrm{~mm}$ in diameter, or $24 \mathrm{~mm}$ from the top to bottom raster line. Since there are 525 lines, a resolving element is taken to be a square $24 / 525 \mathrm{~mm}$ per side. Taking $F=105 \mathrm{~mm}$ and $\tau=1 / 30 \mathrm{~s}$,

$$
I_{L}=I_{T} 76.57 \omega ; \quad \omega \geq 0.01306 \mathrm{rad} / \mathrm{s}
$$

Figure 3 compares this model of the response to moving point sources with measurements made in

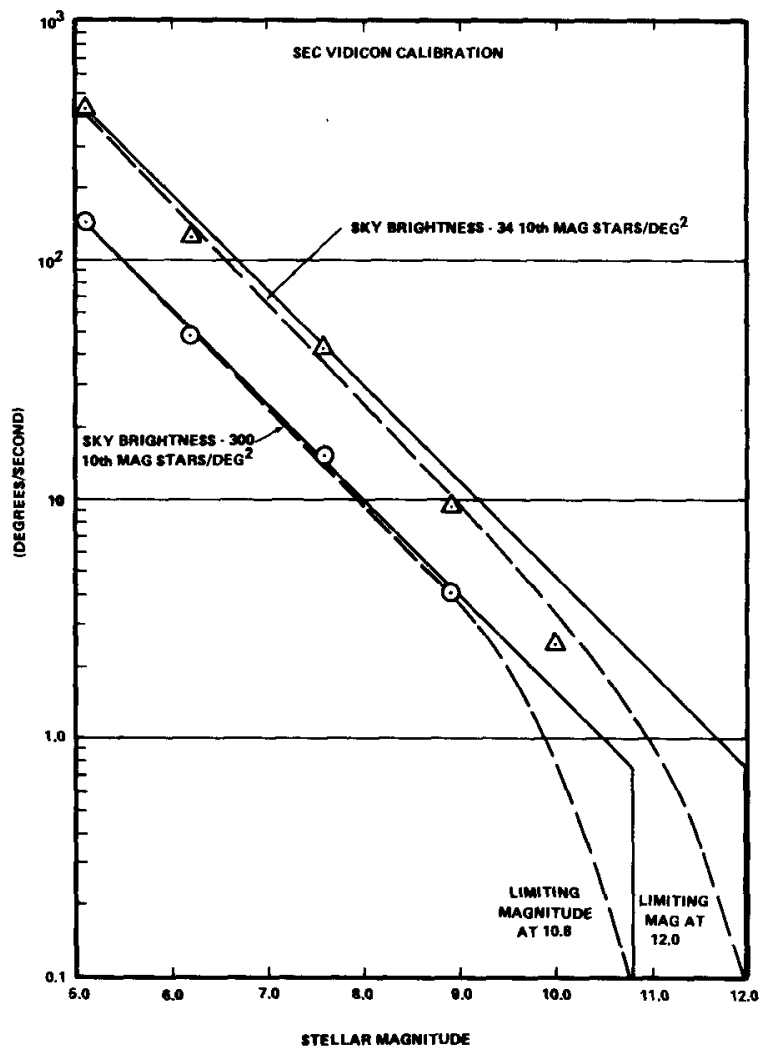

Figure 3.-Response of the SEC Vidicon to moving point images. The solid curves represent the predicted response based on the detection criteria used in the analysis. 
our laboratory. The measurements consisted of projecting point sources onto a mirror mounted on a rate table which reflected the image onto a projection screen. The point source consisted of a precision $50 \mu$ pinhole mounted in a $35-\mathrm{mm}$ slide projector. Neutral density filters were used to vary the image intensity, and the various images were calibrated by a photomultiplier photometer. The background lighting was provided by a small flood lamp with a controllable aperture. The angular rate of the mirror was adjusted for each filter until the image could no longer be detected on the monitor screen.

The agreement between the simple model and the observed results justifies the model. The only discrepancy occurs in the transition at the point where the image spends $\tau$ in a resolving element. Since the image in the experiment is smaller than the resolving element but is not an infinitesimal, some rounding off of the theoretical model in this transition region is to be expected, which, of course, is the observed result.

Since the angular rate

$$
\omega=\frac{v \sin \theta}{r}
$$

and using $r=80 \mathrm{~km}$ typical of meteor heights, equation (15) becomes

$$
I_{L}=\left\{\begin{array}{l}
I_{T} 0.957 v \sin \theta \\
I_{T}
\end{array}\right.
$$

whichever is greater.

\section{RELATION BETWEEN INCIDENT FLUX AND OBSERVED FLUX}

The number of meteors observed per unit area time is given by

$$
\phi=\int_{\text {hemisphere }} d \Omega \cos \theta \int_{0}^{\infty} d v \int_{m T(I T, \theta, v)}^{\infty} d m n_{m v}
$$

where $n_{m v}$ is the directional mass velocity distribution (number per unit area, time, solid angle with masses between $m$ and $m+d m$ and velocities between $v$ and $v+d v)$. The integration is carried out from the threshold $m_{T}$ required to produce an observed signal, which is a function of the threshold response of the system $I_{T}, \theta$, and $v$. Given only the observed $\phi$, clearly there is not sufficient information to solve the integral equation. Several simplifying assumptions are in order. First, to a good approximation the velocity and mass distributions are independent. Second, it will be assumed that the velocities are isotropically distributed, i.e., $n_{m v}$ is independent of $\theta$ and $\phi$. Equation (18) becomes

$$
\phi=2 \pi \int_{0}^{\pi / 2} \cos \theta \sin \theta d \theta \int_{0}^{\infty} n_{v} d v \int_{m T(I T, \theta, v)}^{\infty} n_{m} d m
$$

Even assuming $n_{v}$ is known, there still is insufficient information to define $n_{m}$. However, the fact that the observed $\phi$ can be expressed as a power law, equation (1), suggests the cumulative mass flux $N_{m}$ (number per unit area time with mass $m$ or greater) can be expressed

$$
N_{m}=\pi \int_{m_{T}}^{\infty} N_{m} d m=C m_{T}{ }^{-\alpha}
$$

where $\alpha$ is the population index. With this assumption equation (19) becomes

$\phi=2 C \int_{0}^{\pi / 2} \cos \theta \sin \theta d \theta \int_{0}^{\infty} n_{v} d v m_{T}{ }^{-\alpha}\left(I_{T}, \theta, v\right)$

The threshold mass is from equation (12)

$$
m_{T}=I_{L} 10^{5.297} v^{-4}(\cos \theta)^{-1}
$$

But from equation (17)

$$
m_{T}=\left\{\begin{array}{cc}
I_{T} 10^{5.297} v^{-4}(\cos \theta)^{-1} & \theta \leq \theta_{0} \\
0.957 I_{T} 10^{5.297} v^{-3} \tan \theta & \theta \geq \theta_{0}
\end{array}\right.
$$

where

$$
\theta_{0}=\sin ^{-1}\left(\frac{1}{0.957}\right)
$$

Equation (20) becomes

$$
\begin{array}{rl}
\phi=2 & C I_{T^{-\alpha} 10^{-5.297 \alpha}} \\
& \cdot\left[\int_{0}^{\infty} v^{4 \alpha} n_{v} d v \int_{0}^{\theta_{0}(v)}(\cos \theta)^{1+\alpha} \sin \theta d \theta\right. \\
+ & (0.957)^{-\alpha} \int_{0}^{\infty} v^{3 \alpha} n_{v} d v \\
& \left.\cdot \int_{\theta_{0}(v)}^{\pi / 2}(\cos \theta)^{1+\alpha}(\sin \theta)^{1-\alpha} d \theta\right]
\end{array}
$$


It is convenient to introduce an average $\bar{m}$ defined as the mass of a just detectable meteor having average velocity and $\theta=45^{\circ}$. From equation (21)

$$
\bar{m}=0.957 I_{T} 10^{5.297} v^{-3} \tan 45^{\circ}
$$

Equation (22) becomes

$$
\begin{aligned}
\phi= & \frac{2 C \bar{m}^{-\alpha}}{v^{3 \alpha}}\left[(0.957)^{\alpha} \int_{0}^{\infty} v^{4 \alpha} n_{v} d v\right. \\
& \cdot \int_{0}^{\theta_{0}(v)}(\cos \theta)^{1+\alpha} \sin \theta d \theta+\int_{0}^{\infty} v^{3 \alpha} n_{v} d v \\
& \left.\cdot \int_{\theta_{0}(v)}^{\pi / 2}(\cos \theta)^{1+\alpha}(\sin \theta)^{1-\alpha} d \theta\right]
\end{aligned}
$$

The first integral is the contribution from those meteors moving nearly along the line of sight that remain in a single resolving element for one integration time. Since $\theta_{0}$ is typically $3^{\circ}$, this contribution is small and can be ignored. The integral over $\theta$ in the second integral must be evaluated numerically unless $\alpha$ is an integer. The lower limit $\theta_{0}(v)$ is a function of $v$, however, as may be seen in figure 4. This dependence is not strong for $\alpha \approx 1$. Therefore, an average value of $v=20 \mathrm{~km} / \mathrm{s}$ will be used which yields $\theta_{0}=3^{\circ}$.

The velocity distribution was adopted from the work of Dohnanyi (1966), expressed as

$$
n_{v}= \begin{cases}C_{N} v^{1.6} & 11.2 \leq v \leq 16.6 \\ C_{N} 1.61 \times 10^{7} v^{-4.3} & 16.6 \leq v \leq 72.2\end{cases}
$$

The normalization constant $C_{N}=.001153$. The $\langle v\rangle$ using this distribution is $19.2 \mathrm{~km} / \mathrm{s}$. The weighted avcrage $\left\langle v^{x}\right\rangle /\langle v\rangle^{x}$ is shown in figure 5 .

Equation (24) can be written

$$
\phi=2 \frac{\left\langle v^{3 \alpha}\right\rangle}{\langle v\rangle^{3 \alpha}} I\left(\theta_{0}\right) N_{\bar{m}_{T}}
$$

where $N_{\bar{m}_{T}}$ is the cumulative isotropic mass flux of meteoroids having mass $\bar{m}_{T}$ or greater, $\bar{m}$ is given by equation $(23)$, and $I(\theta)$ is

$$
I\left(\theta_{0}\right)=\int_{\theta_{0}}^{\pi / 2}(\cos \theta)^{1+\alpha}(\sin \theta)^{1-\alpha} d \theta
$$

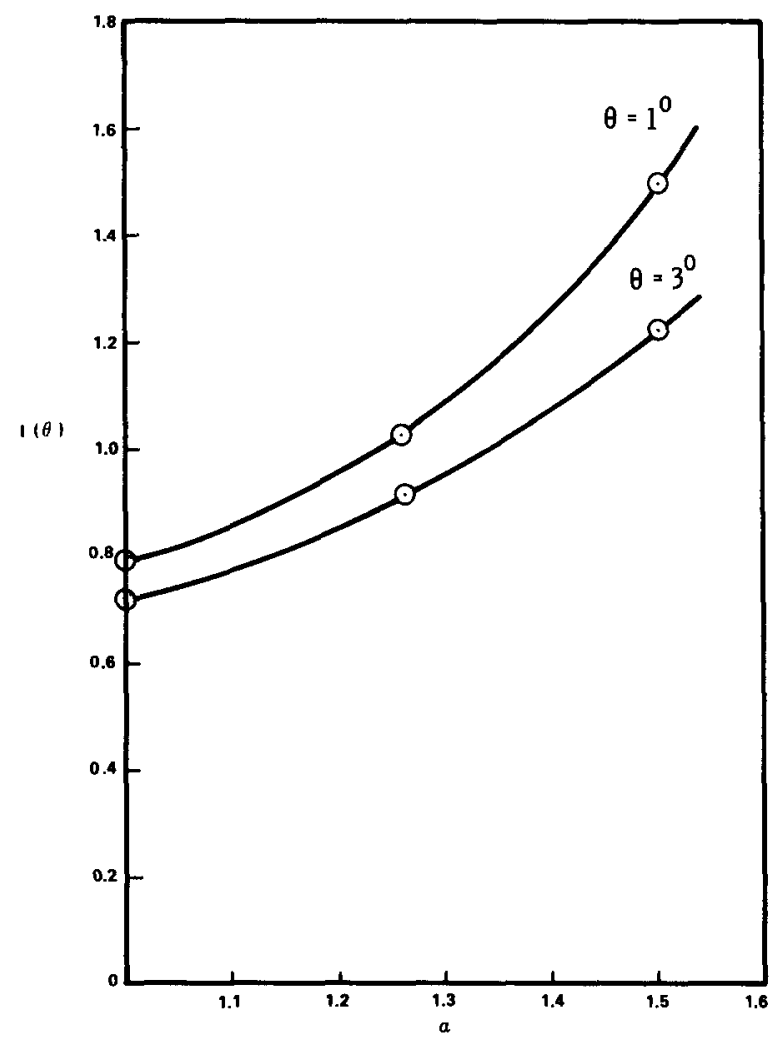

Figure 4.-The value of the integral $I\left(\theta_{0}\right)$ in equation (26) as a function of $\theta_{0}$ and $\alpha$.

Since $I_{T}=10^{-0.4 m_{v}}$ and the observational results were found to be as follows, from equation (1):

$$
\begin{aligned}
\log \phi & =-15.352+0.5053 m_{v} \\
\log \phi & =-15.352-1.263 \log I_{T}
\end{aligned}
$$

Differentiating the $\log$ of equation (22)

$$
\frac{d(\log \phi)}{d\left(\log I_{T}\right)}=-\alpha
$$

From equation (27) $\alpha$ is found to be 1.263. Using this value in figures 4 and 5 ,

$$
\begin{gathered}
\frac{\left\langle v^{3.76}\right\rangle}{\langle v\rangle^{3.76}}=2.48 \\
I(\theta)=0.933
\end{gathered}
$$

Combining equations (23), (26) and (27)

$$
\log N_{\bar{m}_{T}}=-15.352-1.263\left(\log \bar{m}_{T}-1.466\right)
$$




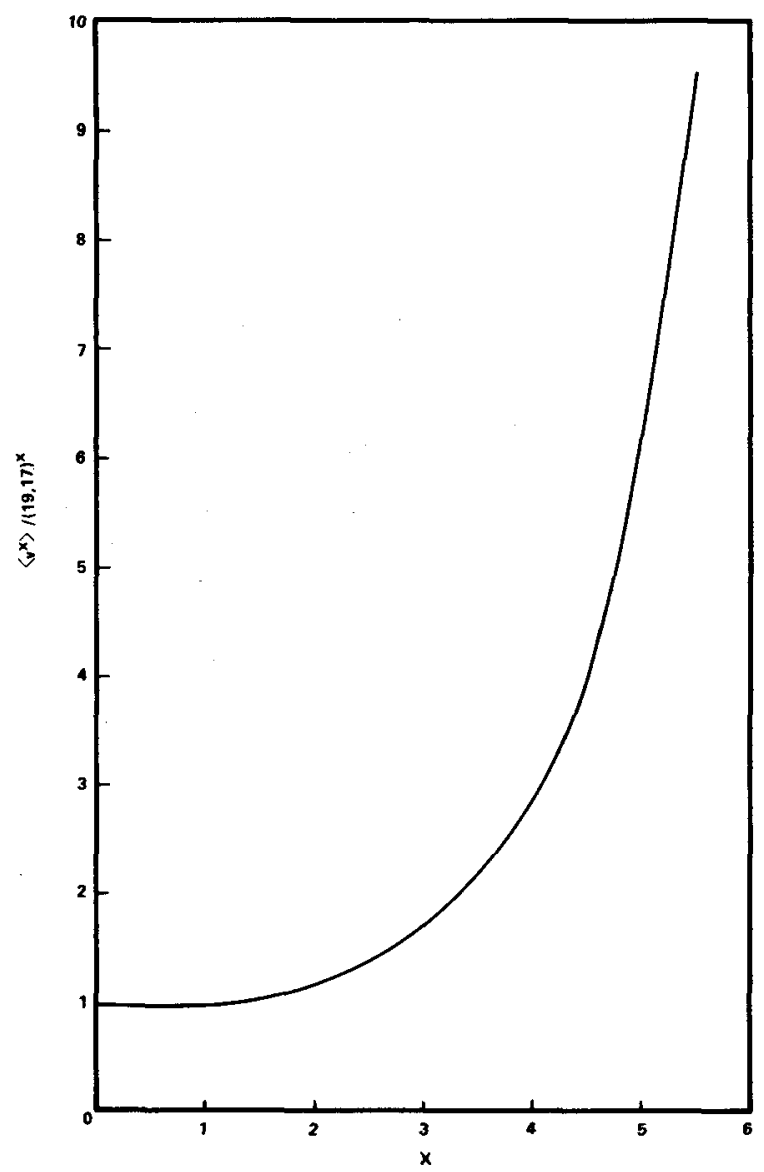

Figure 5.- The value of the moments of the velocity distribution computed from Dohnanyi's velocity distribution.

or

$$
\log N_{\bar{m}_{T}}=-14.24-1.263 \log \bar{m}_{T}
$$

The observed range from $m_{v}=7$ to $m_{v}=10$ corresponds to a mass range of $10^{-1.334}$ to $10^{-2.534} \mathrm{~g}$. The results of equation (28) are compared with the existing distribution of meteors in figure 6 . The result from the July 31 to August 9 expedition is also shown which contains the Perseids and $\delta$-Aquarids. No attempt was made to alter the distributions in velocity and angle to account for the shower component.

\section{COMPARISON WITH OTHER DATA}

Figure 6 shows the relationship of the data obtained in this work with the current meteoroid mass distribution adopted by NASA (Cour-

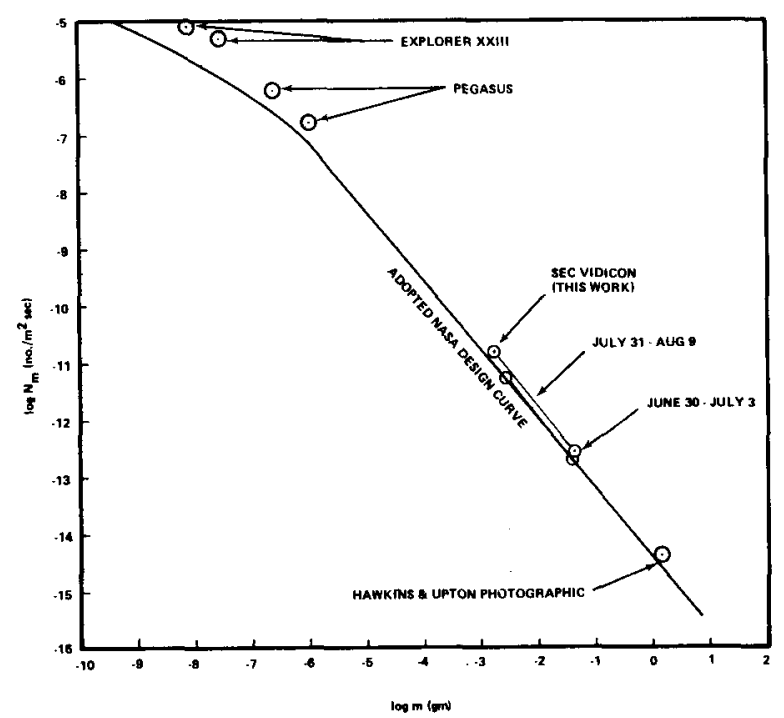

Frgure 6.-Comparison of the results of this study with other work and with the adopted NASA meteoroid environmental design criteria. The observed data represent encounter frequency, whereas the design criteria are weighted to express penetration frequency.

Palais, 1969). Also shown for comparison is the Hawkins and Upton (1958) datum point based on photographic meteors, and the points obtained from the Pegasus (Clifton and Naumann, 1966) and Explorer XXIII penetration experiments (D'Aiutolo, 1965). These data have been analyzed in terms of encounter frequency, i.e., number incident per unit area time on a surface without regard to angle of incidence or velocity. The NASA design curve refers to the number capable of penetrating a surface per unit area time which can just be penetrated by a meteoroid with the specified mass under conditions of normal impact at the average velocity (assumed to be $20 \mathrm{~km} / \mathrm{s}$ ) and average density (assumed to be $0.5 \mathrm{~g} / \mathrm{cm}^{3}$ ). Integrating over the velocity and angular distributions weighted appropriately for penetration mechanics results in the penetration frequency being less than the encounter frequency by a factor of approximately 2 (Naumann, 1966).

The penetration data from Pegasus and Explorer XXIII were analyzed using recent calibration data (Naumann et al., 1969). The discrepancy between these data points and the NASA model is partially due to the difference between encounter frequency and penetration frequency, 
but also includes the departure from linear size scaling in thin metallic targets. Again, the design curve was derived from the actual penetration data, using the conventional penetration formula, and will serve adequately as a design criterion so long as the same formula is used to convert back to penetration results. However, this departure should be considered in developing a true mass distribution.

\section{CONCLUSIONS}

A technique has been developed using the SEC vidicon LLLTV system as a threshold detector for faint meteors to obtain mass flux distribution data in the mass range from 1 to 100 milligrams. The analysis technique is based on peak intensities using the most recent values of luminous efficiency obtained from the Trailblazer measurements. The data are quite consistent with present photographic data at 1 gram and the satellite data at 1 microgram, and tend to confirm the adopted NASA meteoroid model.

It is recognized that these observations represent only one time during the year and may be subject to seasonal variations. Such effects are the object of a current investigation.

\section{REFERENCES}

Ayers, W. G., McCrosky, R. E. And Shao, C. -Y., 1970. Photographic observations of 10 artificial meteors, Smithson. Obs. Spec. Rept. No. 317.

Beyer, R. R., Green, M., and Goetze, G. W., 1966. Point-source imaging with SEC target, in Advances in Electronics and Electron Physics, 22A, Academic Press, New York, 251-260.

Cufton, K. S., And Naumann, R. J., 1966. Pegasus satellite measurements of meteoroid penetration, NASA Tech. Memo X-1316.

Cook, A. F., J ACChiA, L. G., ANd MCCrosky, R. E., 1963. Luminous efficiency of iron and stone asteroidal meteors, Smithson. Contrib. Astrophys., 7, 209-220.

Cour-Palais, B. G., 1969. NASA space vehicle design criteria-environment. Meteoroid environment model-1969. (Near Earth to lunar surface), NASA SP-8013, Supt. of Documents, U.S. Govt. Printing Office, Washington.

D'Aiutolo, C. T., Kinard, W. H., and Naumann, R. J., 1965. Recent NASA meteoroid penetration results from satellites, Smithson. Contrib. Astrophys., 11, 239-251.

Dohnany, J. S., 1966. Bellcomm TR-66-340-1.

Hawkins, G. S., And Upron, E. K. L., 1958. The influx rate of meteors in the Earth's atmosphere, Astrophys. J., 128, 727-735.

JACChIA, L. G., Verniani, F., AND Briggs, R. E., 1967. An analysis of the atmospheric trajectories of 413 precisely reduced photographic meteors, Smithson. Contrib. Astrophys., 10, 1-139.

Naumann, R. J., 1966. Near-Earth meteoroid environment, NASA Tech. Note TN D-3717.

Naumann, R. J., Jex, D. W., And Jonnson, C. L., 1969. Calibration of Pegasus and Explorer XXIII detector panels, NASA Tech. Rept. TR R-321. 